\title{
Development of a miRNA surface- enhanced Raman scattering assay using benchtop and handheld Raman systems
}

Monika Schechinger

Haley Marks

Andrea Locke

Mahua Choudhury

Gerard Cote 


\title{
Development of a miRNA surface-enhanced Raman scattering assay using benchtop and handheld Raman systems
}

\author{
Monika Schechinger, ${ }^{a, *}$ Haley Marks, ${ }^{a}$ Andrea Locke, ${ }^{a}$ Mahua Choudhury, ${ }^{b}$ and Gerard Cote ${ }^{a, c}$ \\ ${ }^{a}$ Texas A\&M University, Department of Biomedical Engineering, College Station, Texas, United States \\ ${ }^{\mathrm{b} T}$ Texas A\&M University, Irma Lerma Rangel College of Pharmacy, College Station, Texas, United States \\ ${ }^{\top}$ Texas A\&M Engineering Experimentation Station, Center for Remote Health Technologies and Systems, College Station, Texas, United States
}

\begin{abstract}
DNA-functionalized nanoparticles, when paired with surface-enhanced Raman spectroscopy (SERS), can rapidly detect microRNA. However, widespread use of this approach is hindered by drawbacks associated with large and expensive benchtop Raman microscopes. MicroRNA-17 (miRNA-17) has emerged as a potential epigenetic indicator of preeclampsia, a condition that occurs during pregnancy. Biomarker detection using an SERS point-of-care device could enable prompt diagnosis and prevention as early as the first trimester. Recently, strides have been made in developing portable Raman systems for field applications. An SERS assay for miRNA-17 was assessed and translated from traditional benchtop Raman microscopes to a handheld system. Three different photoactive molecules were compared as potential Raman reporter molecules: a chromophore, malachite green isothiocyanate (MGITC), a fluorophore, tetramethylrhodamine isothiocyanate, and a polarizable small molecule 5,5-dithio-bis-(2-nitrobenzoic acid) (DTNB). For the benchtop Raman microscope, the DTNB-labeled assay yielded the greatest sensitivity under 532-nm laser excitation, but the MGITC-labeled assay prevailed at $785 \mathrm{~nm}$. Conversely, DTNB was preferable for the miniaturized 785-nm Raman system. This comparison showed significant SERS enhancement variation in response to 1-nM miRNA17 , implying that the sensitivity of the assay may be more heavily dependent on the excitation wavelength, instrumentation, and Raman reporter chosen than on the plasmonic coupling from DNA/miRNA-mediated nanoparticle assemblies. $\odot 2018$ Society of Photo-Optical Instrumentation Engineers (SPIE) [DOI: 10.1117/1.JBO.23.1.017002]
\end{abstract}

Keywords: surface-enhanced Raman spectroscopy; plasmonics; point of care diagnostics; DNA functionalized nanoparticles; microRNA-17; preeclampsia.

Paper 170432PR received Jul. 5, 2017; accepted for publication Dec. 1, 2017; published online Jan. 8, 2018.

\section{Introduction}

Blood biomarker detection has been successfully used for diagnosis in both laboratory and clinical settings. Due to a reduction in system size and cost, along with the discovery of additional biomarkers, commercial devices are emerging for use at the point-of-care (POC). Early detection of human biomarkers can indicate the body's relative state prior to the onset of disease and often before symptoms present, thus underscoring the importance of rapid blood biomarker detection at the POC. The diagnosis of preeclampsia is one potential application of blood biomarker detection. Effective testing would require a POC device that can identify a preeclampsia-associated biomarker as early as the first trimester. Specifically, the detection of epigenetic-miRNA blood biomarkers could be a significant step toward prevention. ${ }^{1}$

Preeclampsia usually develops late in pregnancy during the second and third trimester. Currently, there are no available treatments aside from delivering the babies, which often leads to preterm birth, intrauterine growth-restriction, or sometimes death. Ideally, patients should undergo therapeutic interventions prior to disease onset. Thus, developing methodologies to predict high-risk pregnancy complications prior to conception and early in pregnancy is key. Recently, the interaction between

*Address all correspondence to: Monika Schechinger, E-mail: monikab @tamu .edu genes and environment has emerged as a new frontier for discovering how networks of modified genes can cause several major pathologies, including preeclampsia. An emerging concept within this field suggests that epigenetic-miRNA dysregulation during early pregnancy may lead to preeclampsia. Epigenetic-miRNA changes occur prior to any genetic changes. More importantly, they are reversible, thus making them ideal candidates for therapeutic intervention. Therefore, identifying the epigenetic-miRNA biomarkers early in first trimester can potentially lead to successful treatment of at-risk patients. ${ }^{2,3}$

The current method for diagnosing preeclampsia uses a symptoms-based approach. Symptoms associated with preeclampsia include: elevated blood pressure, protein in the urine, headache, nausea, anxiety, problems with vision, and confusion. ${ }^{2,4,5}$ Generally, these symptoms can be overlooked, since they are commonly reported alongside many different ailments. Thus, the symptoms-based approach for diagnosing preeclampsia is characterized by a lack of specific, indisputable diagnostic criteria even during the second trimester.,

Surface-enhanced Raman spectroscopy (SERS), known for its high sensitivity and capacity for multiplexing analytes, is an emerging molecular sensing and detection platform for applications such as the early diagnosis of preeclampsia. ${ }^{8}$ The technique utilizes plasmonics to target the electromagnetic

$1083-3668 / 2018 / \$ 25.00$ @ 2018 SPIE 
component of the optical signal by combining rough metallic surfaces or noble metal nanoparticles with a surface-associated chemical such as a Raman reporter molecule and high-energy excitation light. ${ }^{9,10}$ Combining SERS with resonant effects from molecular labeling further enhances Raman scattering. ${ }^{11}$ Depending on the absorption properties of the Raman reporter molecule used, the SERS signal can be enhanced through resonance matching, or alternatively, could be completely clouded by background fluorescence. Many Raman reporter molecules can induce additional signal enhancements via resonance Raman. Surface-enhanced resonance Raman scattering occurs when the target molecule has an electronic transition close to or coincident with the Raman excitation wavelength and is aligned with the surface plasmon resonance (SPR) of a nanomaterial bound to the reporter dye. This process typically requires additional consideration when choosing appropriate Raman active dye molecules and nanoparticle dyes optimized for the excitation laser used. ${ }^{12}$

Historically, Raman spectroscopy required the use of large, bulky, and expensive benchtop microscope systems. ${ }^{13}$ Thus, taking advantage of SERS' capabilities in clinical applications requires overcoming the shortcomings associated with these traditional systems along with improving assay reproducibility. ${ }^{14,15}$ Recent developments of handheld and portable Raman devices may facilitate quick and easy on-site medical testing. ${ }^{16}$ However, the results observed using compact Raman spectrometers are often of lower quality compared with the Raman spectra obtained using most benchtop systems. This is due, in part, to compact spectrometers' low-power laser diode sources and lower resolution (but more cost effective) optical components. Though early systems used near-infrared (NIR) wavelengths, systems using shorter, higher energy wavelengths are now available. However, these new systems are typically more expensive or bulky because they require high power sources. Conversely, NIR laser diodes produce lower energy excitation, thus often sacrificing sensitivity, but they require less energy and can be battery operated. In particular, NIR excitation sources are commonly used with biological samples due to their longer sample penetration depths. Further, the longer wavelengths of NIR sources produce less background noise from autoflourescence of biological samples when compared with shorter wavelength lasers. ${ }^{17}$ In contrast, lasers at shorter wavelengths such as $532 \mathrm{~nm}$ produce higher energy Raman scattering, which typically provides a clearer SERS signal when used with most common reporter dyes. However, prolonged exposure of a sample to high-energy (i.e., shorter) wavelengths can result in sample evaporation and photochemical degradation, in addition to stronger autoflourescence from tissue. Therefore, optimizing enhancement factors that create a strong and consistent Raman signal is key to compensating for the lower optical resolution associated with portable and handheld Raman systems.

\section{Materials and Methods}

\subsection{Overview of Methods}

The influence of excitation wavelength on the miRNA-17 SERS assay signal was initially investigated. Lasers with excitation wavelengths at 532 and $785 \mathrm{~nm}$ were chosen to compare signal enhancement from DNA-coated silver nanoparticles conjugated with Raman reporter molecules. Silver nanoparticles were chosen due to their ability to provide surface oxidation and enhanced Raman scattering. ${ }^{18}$ Additionally, the larger extinction coefficient of silver nanoparticles when compared with gold nanoparticles provides larger SERS enhancements. In parallel, the comparative intensity of the assay's SERS signal using different types of photoactive Raman reporter molecules was assessed. Highly polarizable small molecules such as 4-mercaptobenzoic acid, ${ }^{19}$ 5,5-dithio-bis-(2-nitrobenzoic acid) (DTNB), ${ }^{20}$ and 4-mercaptopyridine ${ }^{21}$ are commonly used as reporter dyes since they provide simple, distinct, and readily recognizable Raman spectra. Fluorophores are among the most common dye molecules used in sensing and are often used in Raman spectroscopy because they are widely available. However, their overwhelming emissions can sometimes interfere with the Raman signal and cause a large degree of background signal when not properly quenched. ${ }^{22}$ Finally, the use of synthetic

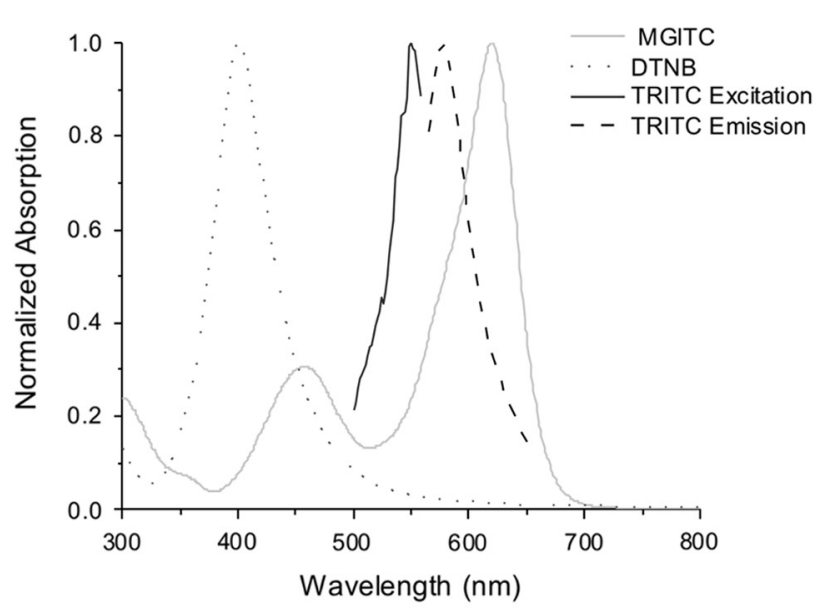

(a)

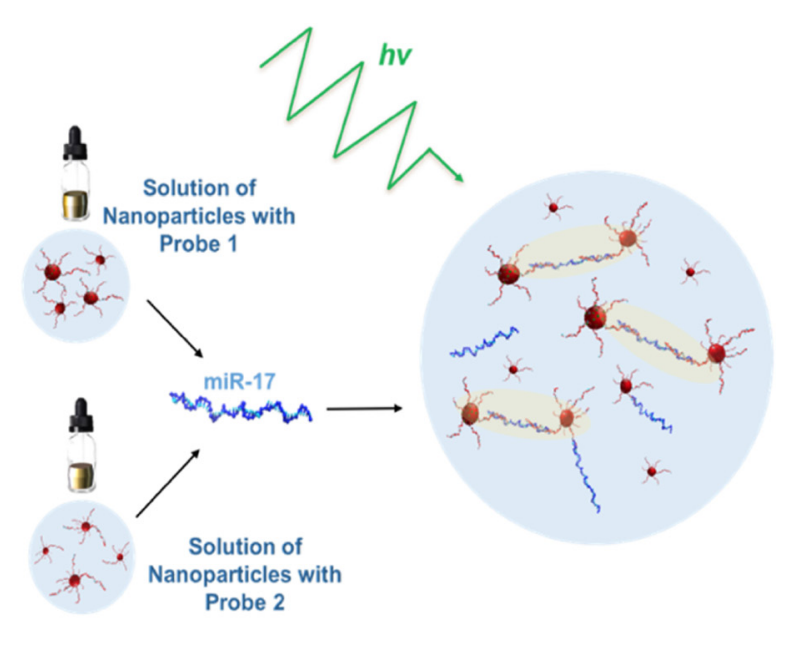

(b)

Fig. 1 (a) Comparison of absorption spectra of the three Raman reporter molecules (MGITC, DTNB, and TRITC) and the fluorescent excitation and emission spectra for the fluorophore, TRITC; (b) a graphic representation of the assay. 


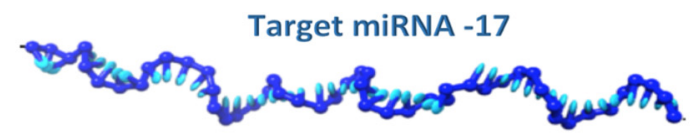

3'GAU GGA CGU GAC AU UCG UGA AAC ${ }^{\prime}$

3' THIOL HEG CTA CCT GCA CTG 5' 3'TA AGC ACT TTG HEG THIOL 5'
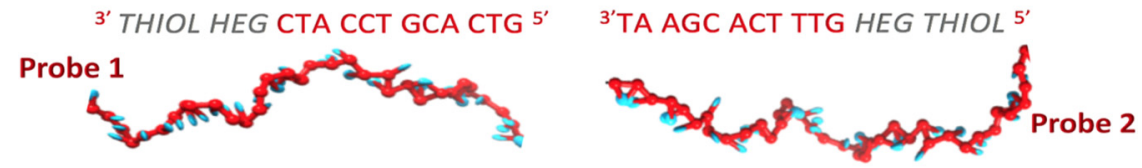

Fig. 2 Schematic of two modified DNA probes selected for hybridization to the target analyte miRNA-17.

nonfluorescent dyes-most often those with triphenylmethane backbones such as crystal violet, victoria blue, and malachite green - has become increasingly popular since it avoids fluorescence emission and offers absorbance overlap with common Raman excitation laser lines. For our study, three types of molecules commonly used as Raman reporters were investigated, namely: (1) a chromophore, malachite green isothiocyanate (MGITC), with absorption maxima at $620 \mathrm{~nm}$, (2) a fluorophore, tetramethylrhodamine isothiocyanate (TRITC), with absorption maxima at $550 \mathrm{~nm}$, and (3) a Raman active molecule, DTNB, with absorption maxima at $402 \mathrm{~nm} \cdot{ }^{12,23}$ As a fluorophore, TRITC also features a characteristic emission wavelength at $576 \mathrm{~nm}$ [Fig. 1(a)]. ${ }^{24}$

After comparing the nonfluorescent chromophore, Ramanactive molecule, and fluorophore, the molecules that showed adequate signal enhancement with the benchtop system were then examined using a handheld Raman device with an excitation laser at $785 \mathrm{~nm}$.

Specifically, this study used a "turn on" sandwich nanoparticle assay developed for detecting nanomolar levels of microRNA-17 using SERS [Fig. 1(b)]. ${ }^{25}$ Silver nanoparticles were functionalized by immobilizing modified oligonucleotide strands at the surface of the nanoparticle. Two independent thiolmodified strands of PEGylated oligonucleotides were designed, with each strand complimentary to half of the target miRNA (Fig. 2) ${ }^{26,27}$ Probe 1 was designed to bind to the five prime $\left(5^{\prime}\right)$ end of the target, whereas probe 2 was constructed to bind to the second half of the target at the three prime $\left(3^{\prime}\right)$ end, with a hexaethylene glycol (HEG) spacer between the end of the DNA probe and the thiol modification. ${ }^{28-30}$ Nanoparticles were functionalized entirely with either probe 1 or probe 2 , and then mixed at a 1:1 volume ratio. If miRNA-17 is present, the target strand is oriented in between two nanoparticles and hybridization to the target draws the nanoparticles close to one another, allowing plasmons to couple and create an area of high energy known as a "hotspot." $31-33$ The high affinity of the nanoparticle probes with the target analyte causes a nanoparticle to simultaneously bind with multiple targets, creating an aggregate or matrix of interconnected units of nanoparticles bound with miRNA. This sandwich-type assay is common among detection assays in which an enhancement in the SERS signal is observed when the target is present. ${ }^{10,34}$

\subsection{Instrumentation}

Benchtop Raman spectra were obtained using a 10x ThermoScientific DXR Raman confocal microscope (Thermo Fisher Scientific; Waltham, Massachusetts) with 900 and $830 \mathrm{~g} / \mathrm{mm}$ gratings for excitation lasers with wavelengths of 532 and $785 \mathrm{~nm}$, respectively. To capture the spectra, a thermoelectrically cooled charged-coupled device (CCD) camera was used in combination with the Raman microscope. Additional handheld Raman spectra were collected using the IDRaman mini 2.0 system (Ocean Optics; Florida). Table 1 shows a summary of the defined optical parameters used for each Raman system as well as a brief overview of their sensing capabilities. $^{35-38}$

The nanoparticles were characterized using a Zetasizer Nano ZS90 (Malvern, United Kingdom) as well as a transmission electron microscope (TEM) (FEI Company; Hillsboro,

Table 1 Summary of optical specifications of the Raman system tested (benchtop ${ }^{32}$ and handheld ${ }^{33,34,38}$ ).

\begin{tabular}{|c|c|c|c|c|c|c|c|}
\hline & $\begin{array}{c}\text { Excitation } \\
\text { Wavelength }\end{array}$ & Raman Shift & $\begin{array}{c}\text { Spectral } \\
\text { Resolution }\end{array}$ & $\begin{array}{c}\text { Power at } \\
\text { Source }\end{array}$ & $\begin{array}{l}\text { Power at } \\
\text { Sample }\end{array}$ & Spot Size & $\begin{array}{l}\text { Integration } \\
\text { Time }\end{array}$ \\
\hline \multicolumn{8}{|l|}{ Benchtop } \\
\hline \multicolumn{8}{|c|}{ Thermo Scientific } \\
\hline \multirow[t]{2}{*}{ DXR Raman } & 532 & 50 to $1800 \mathrm{~cm}^{-1}$ & $2 \mathrm{~cm}^{-1}$ & $24 \mathrm{~mW}$ & $10 \mathrm{~mW}$ & $10 \mu \mathrm{m}$ & $1.0 \mathrm{~s}$ \\
\hline & 780 & & & $80 \mathrm{~mW}$ & $24 \mathrm{~mW}$ & & \\
\hline
\end{tabular}

Handheld

Ocean Optics

IDRaman mini

785

400 to $2300 \mathrm{~cm}^{-1}$

18 to $20 \mathrm{~cm}^{-1}$

$100 \mathrm{~mW}$

$50 \mathrm{~mW}$

$2.5 \mathrm{~mm}$

$100 \mathrm{~ms}$ 
Oregon) to determine their surface charge (zeta potential) and diameter. The absorption characteristics of the nanoparticles were determined using an Infinite ${ }^{\circledR} 200$ Pro multimode microplate reader (Tecan, Switzerland).

\subsection{Materials}

The target miRNA-17 oligonucleotide (sequence: 5'CAA AGU GCU UAC AGU GCA GGU AG3') was synthesized by Integrated DNA Technologies (IDT). The two half-compliment strands of modified single-stranded DNA (ssDNA), sensing probe 1 (sequence: 5'THIOL HEG CTA CCT GCA CTG3') and sensing probe 2 (sequence: 5'TAA GCA CTT TG HEG THIOL3'), were also synthesized by IDT. MGITC and tetramethylrhodamine isothiocyanate (TRITC) dyes were purchased from Invitrogen (United Kingdom). The remaining chemicals and reagents, including the DTNB dye, were procured from Sigma Aldrich (USA/UK). Hydroxylamine silver nanoparticles were synthesized using an adaptation of the rapid, room temperature synthesis method developed by Leopold and Lendl. ${ }^{18}$ The concentration of each sample was calculated using BeerLambert's Law: $A=c \varepsilon_{\lambda} l$.

\subsection{Assay Functionalization}

The modified oligonucleotide probes were purchased in a disulfide form and were subsequently reduced to contain thiol "sticky-ends" and washed before being immobilized onto the nanoparticle surface. To ensure a consistent degree of surface functionalization, the molar ratio of immobilized oligonucleotides added per nanoparticle was set at 1000:1 and the calculated volume of oligonucleotides [24.6 $\mu \mathrm{L}$ of probe $1(34.9 \mu \mathrm{M})$; $41.8 \mu \mathrm{L}$ of probe $2(20.6 \mu \mathrm{M})]$ was added to one of two samples containing $1 \mathrm{~mL}$ of nanoparticles. Each sample contained nanoparticles functionalized entirely with either probe 1 or probe 2 .
The samples were stored separately at room temperature to ensure that neither sample contained a combination of the two probes. Following the addition of the oligonucleotides, the samples were left to react overnight on a shaker table (VWR mixer vortex). After shaking, $60 \mu \mathrm{L}$ of $250-\mu \mathrm{M}$ citrate $\mathrm{HCl}$ solution (pH 3.0) was added to each sample to expedite the reaction, ${ }^{39}$ and the samples reacted for an additional $20 \mathrm{~min}$ while shaking. Next, each sample of now-functionalized silver nanoparticles was centrifuged for $20 \mathrm{~min}$ at $3.3 \mathrm{rcf}$ (relative centrifugal force) to wash away any unconjugated oligonucleotides. For both samples, the resultant supernatant was removed and discarded without disturbing the pellet at the bottom of the tube. Pellets were then resuspended in phosphate buffered saline (PBS) buffer (0.1 M, pH 7.4).

\subsection{Assay Labeling Using Raman Reporter Dyes}

Following nanoparticle functionalization with either probe 1 or probe 2 , the solution of nanoparticles conjugated with probe 1 was labeled using one of the three different types of Raman reporter dyes (MGITC-sample a, DTNB-sample b, and TRITC-sample c). Only nanoparticles functionalized with probe 1 were labeled. No dye was added to nanoparticles functionalized with probe 2 . Using the stock concentration of each dye, the amount of dye was calculated to achieve an ideal ratio of 1000 dye molecules per one nanoparticle. This calculated volume $[85.9 \mu \mathrm{L}$ of dye $(10 \mu \mathrm{M})]$ was then added to samples containing $1 \mathrm{~mL}$ of nanoparticles functionalized with probe 1 . The samples were then shaken for $1 \mathrm{~h}$ before being left overnight at room temperature. Samples were washed by centrifuging the solution for $20 \mathrm{~min}$ at $7000 \mathrm{rpm}$ to remove any excess unconjugated dye. The resultant supernatant was discarded and the nanoparticle pellets were resuspended in PBS buffer at a $\mathrm{pH}$ of 7.4 .
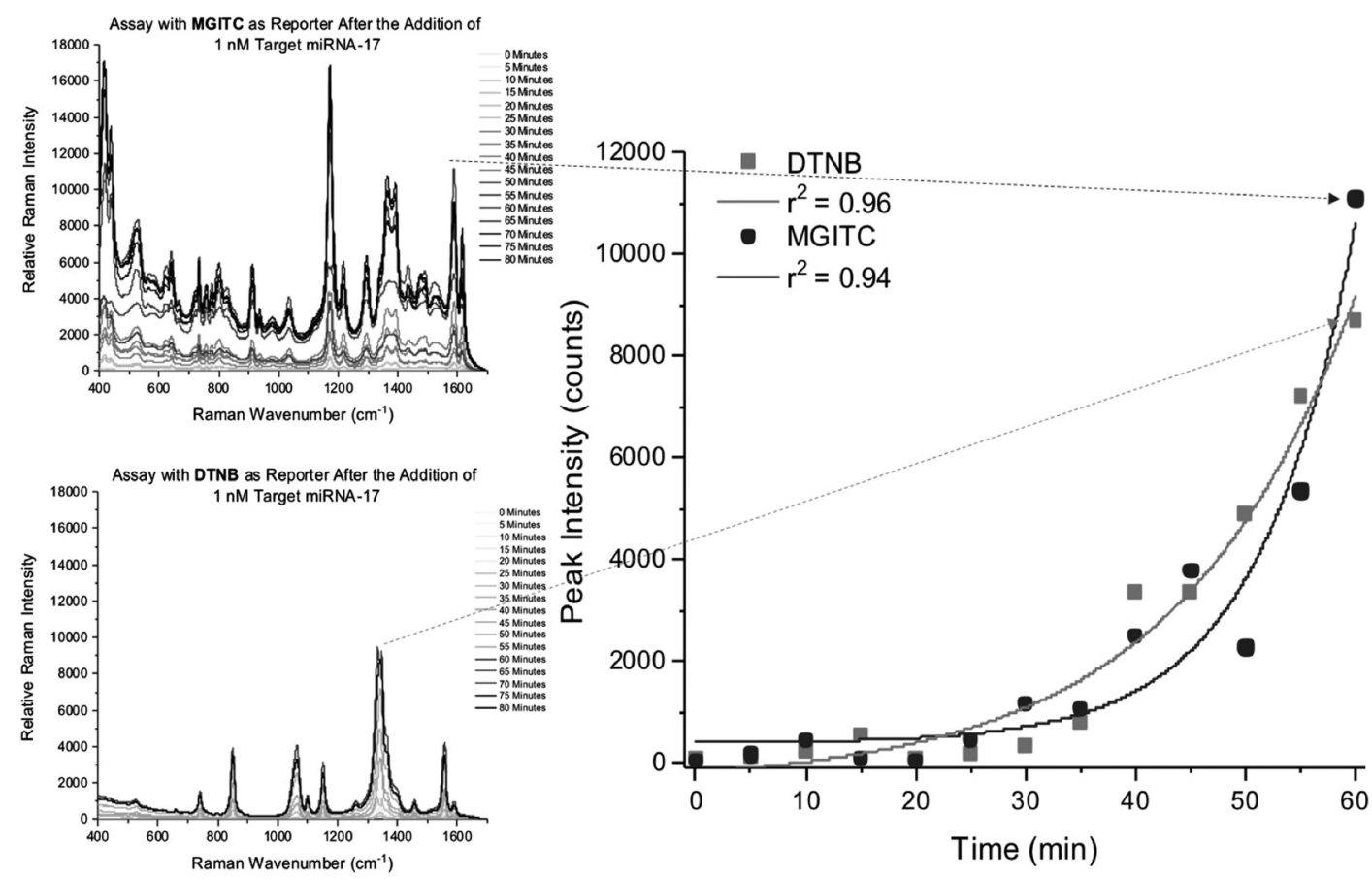

Fig. 3 The change in relative Raman intensity with respect to time after the addition of 1 -nM target miRNA-17. 


\subsection{Assay Analysis Using a Laboratory Benchtop Raman Microscope}

The different variations of the assay (sample assay A with MGITC; sample assay B with DTNB; and sample assay C with TRITC) were loaded separately into a black Greiner 384-well plate and examined using the confocal benchtop Raman microscope. The wells were filled to their maxima volumes of $30 \mu \mathrm{L}$. Equal volumes of nanoparticles conjugated with probe 1 and probe 2 were added to each well. The remaining $10-\mu \mathrm{L}$ volume was composed of either PBS or 1-nM target miRNA-17 suspended in PBS. Wells without miRNA-17 were designated as control samples and used to compare the sensitivity for each embodiment of the assay. Target miRNA-17 was suspended in PBS at a concentration of $1 \mathrm{nM}$. This concentration remained constant among all experimental samples. A pipette was used to mix the resultant solution thereby allowing the target to be dispersed throughout the nanoparticle detection assay. Prior tests showed that for $30 \mu \mathrm{l}$ of sample, the SERS intensity gradually increased until it reached steady maxima intensity after $1 \mathrm{~h}$. Therefore, the sample were placed in the measurement plate and set aside for $1 \mathrm{~h}$ to allow for optimal aggregate formation (Fig. 3). Subsequent SERS analysis was performed using a Thermo Scientific DXR Raman Microscope at excitation wavelengths of 532 and $785 \mathrm{~nm}$.

The nanoparticle assay was tested further to determine its specificity for miRNA-17 and to identify any possible changes in the baseline signal due to the addition of a noncomplimentary target strand. The noncomplimentary strand used was miRNA34a-3p (Sequence: 5'CAA UCA GCA AGU AUA CUG $\left.\mathrm{CCU}^{\prime}\right)$. Once again, two separate samples solutions were created containing either the nanoparticle assay in PBS or the nanoparticle assay combined with $10 \mu \mathrm{L}$ of a $1-n M$ solution of miRNA-17. Additionally, a third sample was created by mixing $20 \mu \mathrm{L}$ of the nanoparticle assay $(10 \mu \mathrm{L}$ of probe 1 solution and $10 \mu \mathrm{L}$ of probe 2 solution) with $10 \mu \mathrm{L}$ of a $1-\mathrm{nM}$ solution of miRNA-34a-3p in PBS. Once synthesized, all three samples were left to react for $1 \mathrm{~h}$. Using an excitation laser at $532 \mathrm{~nm}$, the Raman signal for each of the samples was then observed with the benchtop Raman microscope.

\subsection{Assay Analysis Using a Handheld Raman System}

The various sample assays were loaded into 2-mL glass scintillation vials for analysis using a handheld device. A sample

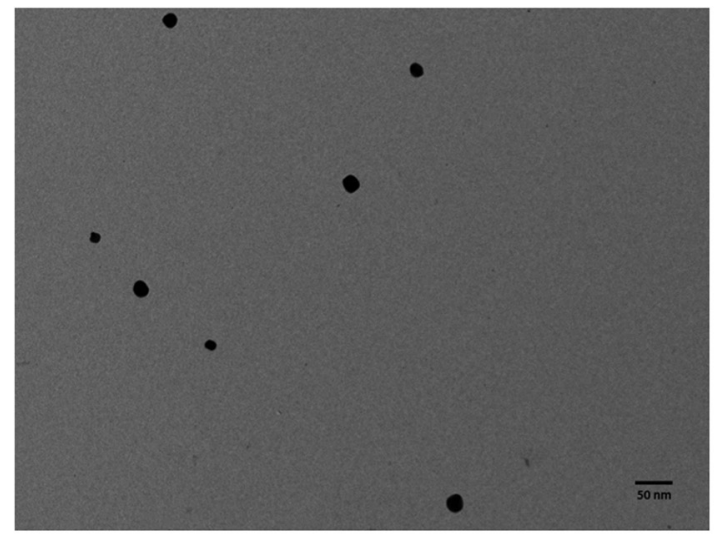

(a)

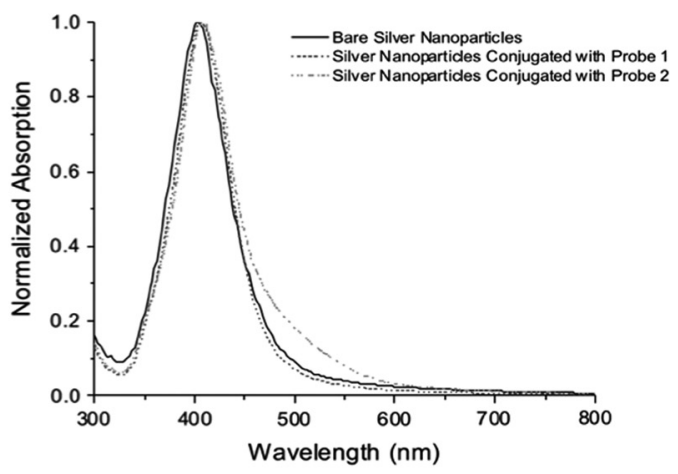

(b)

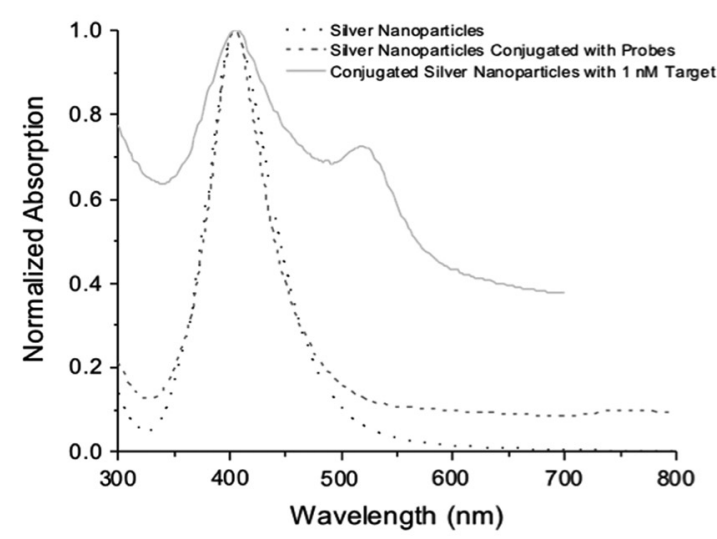

(c)

Fig. 4 (a) TEM images of silver nanoparticles; (b) absorption spectra of silver nanoparticles conjugated with probe 1 and probe 2; and (c) the extinction spectra for bare unconjugated silver nanoparticles, a combined solution of silver nanoparticle conjugated with either probe 1 or probe 2 , and an aggregated combined solution resulting from the addition of the target analyte, miRNA-17. 
volume of $600 \mu \mathrm{L}$ was used for each vial, with each containing: $200 \mu \mathrm{L}$ of silver nanoparticles functionalized with a Raman reporter and probe $1,200 \mu \mathrm{L}$ of silver nanoparticles with probe 2, and $200 \mu \mathrm{L}$ of either PBS or target suspended in PBS. The concentration of the miRNA-17 in PBS was $1 \mathrm{nM}-$ the same molar ratio used with the benchtop Raman system. Similarly, the subsequent reaction time necessary for maxima SERS intensity was also denoted as $1 \mathrm{~h}$. These assays were analyzed using the IDRaman mini handheld Raman system by Ocean Optics. The sample vials were loaded directly into a specially designed chamber, which used an excitation laser at $785 \mathrm{~nm}$. The IDRaman mini used the maxima laser power at level 5 (100 $\mathrm{mW}$ at the source and $50 \mathrm{~mW}$ at the sample), ${ }^{37,40}$ with an exposure time of $100 \mathrm{~ms}$.

\section{Results and Discussion}

\subsection{Characterization Data}

TEM images showed that the diameter of the synthesized silver nanoparticles was $29 \mathrm{~nm}(\mathrm{SDEV} \pm 2 \mathrm{~nm})$ [Fig. 4(a)]. Experimentally, this diameter was confirmed via UV-vis spectroscopy measurements. The maximum absorbance intensity was located at a peak wavelength of $405 \mathrm{~nm}$ [Fig. 4(b)], which corresponds to $\sim 30 \mathrm{~nm}$ and an extinction coefficient of $\left(1.45 \times 10^{10} \mathrm{M}^{-1} \mathrm{~cm}^{-1}\right)$. Based on the values for extinction coefficient and absorbance maximum at $405 \mathrm{~nm}$, the silver nanoparticle concentration was calculated using Beer-Lambert's
Law $\left(A=c \varepsilon_{\lambda} l\right)$ as $81.6 \mathrm{nM}$. The solution of silver nanoparticles was used to synthesize individual stock solutions of nanoparticles conjugated with probe 1 and nanoparticles with probe 2.

Following conjugation, the absorption spectra were collected for both stock solutions and used to confirm successful nanoparticle conjugation with probes [Fig. 4(b)]. In both cases, the absorption maximum increased by $2 \mathrm{~nm}$ indicating successful conjugation of the nanoparticles with probe. The absorption spectra were also used to verify colloid stability and the lack of aggregate formation. No change in nanoparticle size was concluded based on the minimal shift in the absorption maximum associated with conjugation. Furthermore, the nonexistence of secondary peaks confirms the absence aggregates that cause a shift in the absorption maximum toward higher wavelengths and the emergence of secondary peaks.

The assay was also tested to determine its capability to form aggregates in the presence of miRNA-17. The formation of aggregates is the underlying factor, which provides an increase in relative Raman intensity when the target is present, as it facilitates plasmonic coupling. The absorption spectra of unconjugated silver colloid and DNA-functionalized silver nanoparticles were obtained, as was a combined assay solution of functionalized nanoparticles assembled with miRNA-17 [Fig. 4(c)]. The samples of plain and functionalized nanoparticles produced a single peak at $\sim 405 \mathrm{~nm}$ corresponding to its localized surface plasmon resonance (LSPR). The combined

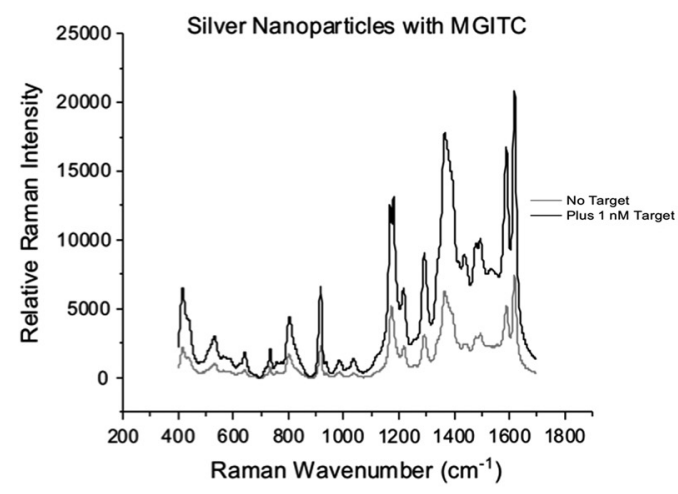

(a)

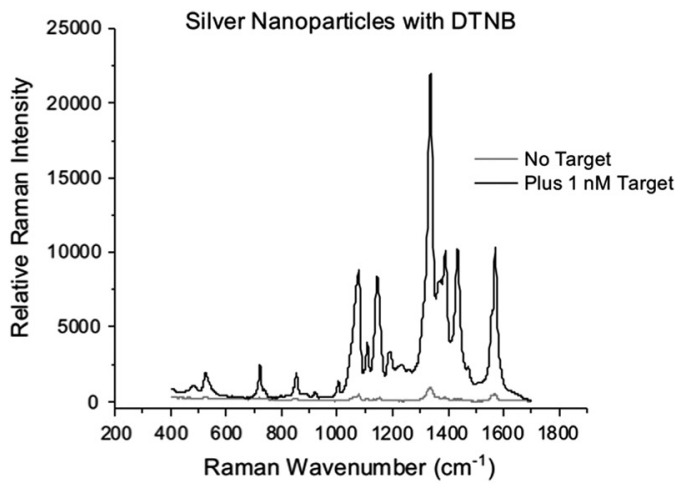

(b)

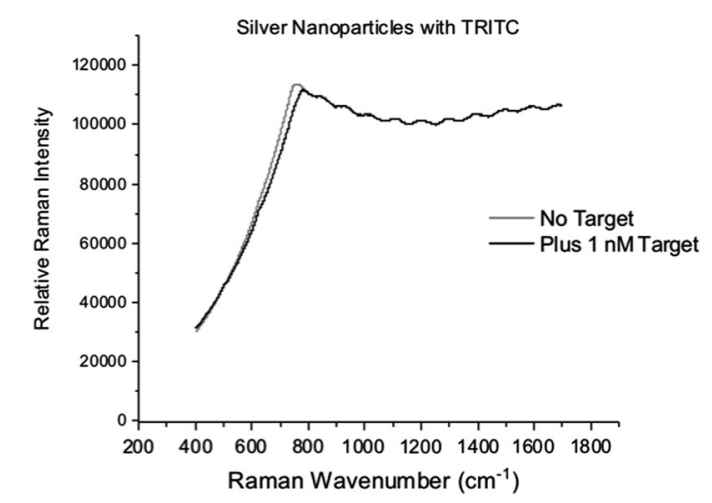

(c)

Fig. 5 The average Raman spectra obtained using an excitation wavelength of $532 \mathrm{~nm}$ from various combinations of silver nanoparticles and photoactive dyes including: (a) MGITC, a chromophore; (b) DTNB, a Raman active molecule; and (c) TRITC, a fluorophore. 
assay with miRNA-17 provided absorption spectra with two LSPR peaks, one at $\sim 405 \mathrm{~nm}$ representing the unbound probe nanoparticles and a second at $\sim 520 \mathrm{~nm}$ indicating the plasmonic coupling that occurs when particles are brought in close proximity with one another by the target miRNA.

\subsection{Comparison of Different Raman Reporter Molecules Using a Benchtop Raman System}

The benchtop Raman system was first used to investigate the role of excitation wavelength in providing SERS signal enhancement. A diode-pumped, solid-state green laser, which produces an excitation wavelength in the visible spectrum $(532 \mathrm{~nm})$, was first used in combination with the benchtop Raman system to examine the SERS spectra for the three Raman reporter molecules using the miRNA-17 assay developed (Fig. 5).

The SERS signal was significantly different for the three Raman reporter molecules when excited using the same laser source. For the 532-nm excitation wavelength, the assay containing DTNB produced a slightly higher maxima signal after the addition of 1-nM target miRNA-17 compared with the assay containing MGITC, thus indicating increased sensitivity for DTNB. With respect to the TRITC reporter assay, employing a 532-nm laser [Fig. 5(c)] caused the highest relative signal intensity of all reporter molecules and saturated the CCD- even at the lowest laser power setting $(0.1 \mathrm{~mW})$. The signal using a 532-nm laser was overwhelmingly strong due to the broad fluorescent emission of TRITC centered at $576 \mathrm{~nm}$.

The excitation laser was then replaced with a red frequencystabilized single mode diode laser, which produced an excitation wavelength in the NIR, at $785 \mathrm{~nm}$. The SERS signal was once again collected for each Raman reporter molecule using the assay specific to miRNA-17 (Fig. 6). The use of an NIR excitation laser at $785 \mathrm{~nm}$ produced Raman spectra similar to those obtained using the $523 \mathrm{~nm}$ laser. The three assays containing the same concentration of DNA-functionalized silver nanoparticles tagged with the three different Raman reporter dyes were then compared (Fig. 6). This time, silver nanoparticles labeled with MGITC produced the highest Raman intensity when $1 \mathrm{nM}$ of the target miRNA-17 was present. In the presence of the target, the MGITC assay demonstrated an observable increase in the relative Raman intensity. Silver nanoparticles labeled with DTNB still produced signal enhancement both with and without the presence of the target, as was the case with the visible 532$\mathrm{nm}$ source. In the presence of the target, both assays containing MGITC and DTNB showed an observable increase in the relative Raman intensity, though the sensitivity of the MGITC assay was significantly higher. No enhancement was observed for silver nanoparticles labeled with TRITC before the addition of the target miRNA. The spectrum associated with TRITC was visible only after the addition of the target.

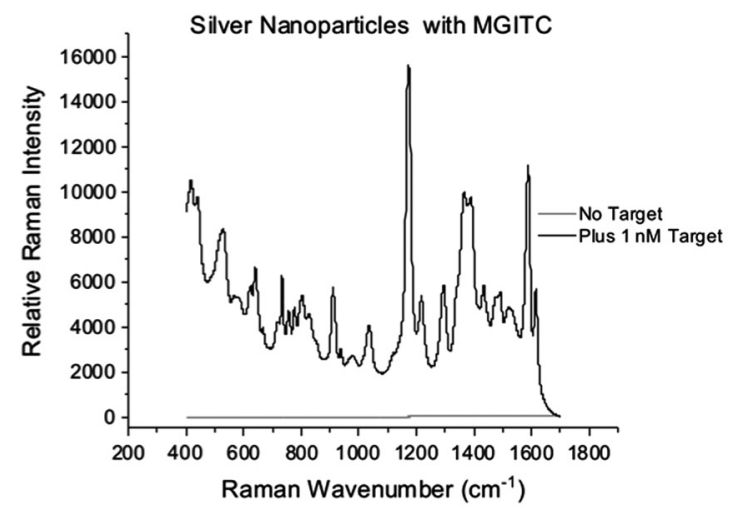

(a)

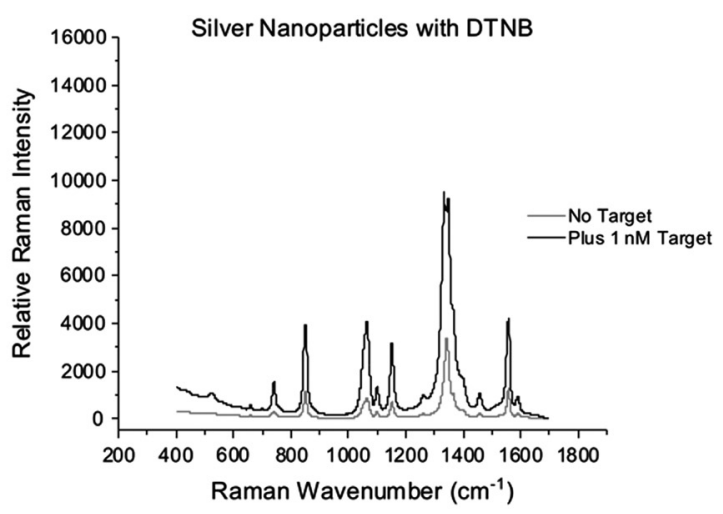

(b)

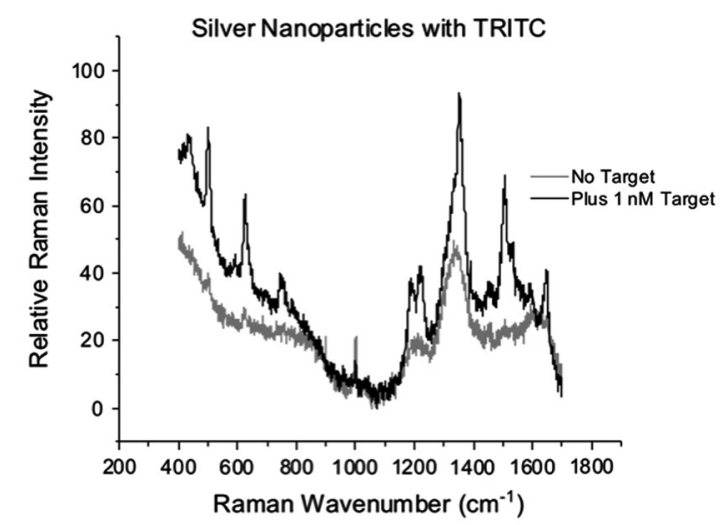

(c)

Fig. 6 The average Raman spectra obtained using an excitation wavelength of $785 \mathrm{~nm}$ from various combinations of silver nanoparticles and photoactive dyes including: (a) MGITC, a chromophore; (b) DTNB, a Raman active molecule; and (c) TRITC, a fluorophore. 
A quick comparison of the SERS spectra collected using the laser at 532 and $785 \mathrm{~nm}$ revealed that MGITC and DTNB were effective in sensing for miRNA-17 for both excitation wavelengths. The use of TRITC as a Raman reporter molecule proved ineffective at both wavelengths due to its inability to provide a discernable signal in the presence of miRNA-17. While MGITC and DTNB were effective at both excitation wavelengths, a noticeable increase in the overall signal intensity was visible when the 532-nm laser was used. The maxima signals observed for both MGITC [Fig. 5(a)] and DTNB [Fig. 5(b)] were almost 10,000 counts greater than those observed using an excitation wavelength of $785 \mathrm{~nm}$ [Figs. 6(a) and 6(b)]. This significant rise in signal indicates the formation of more resonant plasmons within the assay when near the excitation wavelength of $532 \mathrm{~nm}$.

The formation of aggregates occurred because DNA-miRNA hybridization facilitated SERS by combining neighboring plasmons to produce high-energy "hot spots," which caused a resultant red-shift in the LSPR. ${ }^{10,26,41}$ With the visible $(532 \mathrm{~nm})$ source benchtop system, the jump in SERS intensity observed using DTNB yielded the greatest sensitivity. Conversely, the jump in SERS intensity observed using DTNB with the NIR benchtop system was less significant compared with that of the control. Instead, MGITC was the most dramatic and distinguishable, thus denoting MGITC as a seemingly favorable option when using 785-nm laser. These results imply that if the excitation wavelength is increased (red shifted), the Raman reporter molecule should be selected such that its absorbance also undergoes a redshift to avoid losses in the sensitivity of the overall assay system.

The spectra collected were further analyzed by examining the maxima relative Raman intensity using characteristic peaks specific to each Raman reporter. The SERS peak at $1586 \mathrm{~cm}^{-1}$, assigned to the stretching and bending of an in-plane ring, ${ }^{42}$ was chosen for evaluating the assay labeled with MGITC. The strong peak at $1343 \mathrm{~cm}^{-1}$, which corresponds to the symmetric $\mathrm{N}-\mathrm{O}$ stretching of the nitro group, ${ }^{43-45}$ was used to analyze the nanoparticle assay labeled with DTNB. The assay constructed using nanoparticles labeled with the fluorescent molecule did not produce observable Raman spectra inherent to TRITC. It was thus eliminated from further analysis since the lack of discernable spectra made it difficult to assign a characteristic peak for additional examination. The maxima relative Raman intensity shown in Fig. 7(a) was defined by Raman intensity observed, whereas Fig. 7(b) shows the relative Raman intensity at 1586 and $1343 \mathrm{~cm}^{-1}$ for the two useful Raman reporter molecules, MGITC and DTNB. The values seen in Fig. 7 correspond to the average of five spectra with error bars of one standard deviation. Meanwhile, each spectrum used is the average of 10 consecutive $1 \mathrm{~s}$ scans.

The 532-nm laser resulted in the greatest signal enhancement for all three dyes (Fig. 7). MGITC provided signal enhancement for spectra taken both with and without $1 \mathrm{nM}$ of target miRNA17 but produced higher enhancement with the target. The addition of miRNA-17 caused an increase in signal approximately equal to 3.5 times the initial signal intensity observed without miRNA-17 [Fig. 7(b)]. Signal enhancement was much greater in the presence of target using the assay labeled with DTNB for $532 \mathrm{~nm}$. In the case of DTNB, the change in signal was over 16-fold. Unlike MGITC, DTNB produced relatively minor signal enhancement when the target was not present due to the lack of resonate localized surface plasmons. In turn, this resulted in a baseline spectrum at low relative Raman intensities. When combined with the 785-nm laser, silver nanoparticles labeled with MGITC produced the most drastic increase in intensity as compared with nanoparticles labeled with DTNB, which produced the greatest change in intensity when used with the 532-nm laser.

The difference in relative Raman intensity for the assay with DTNB at 532-nm excitation and the assay with MGITC at 785-nm excitation indicates a potential to sense precise target concentrations. To achieve the greatest signal enhancement, researchers typically choose a dye with an excitation wavelength

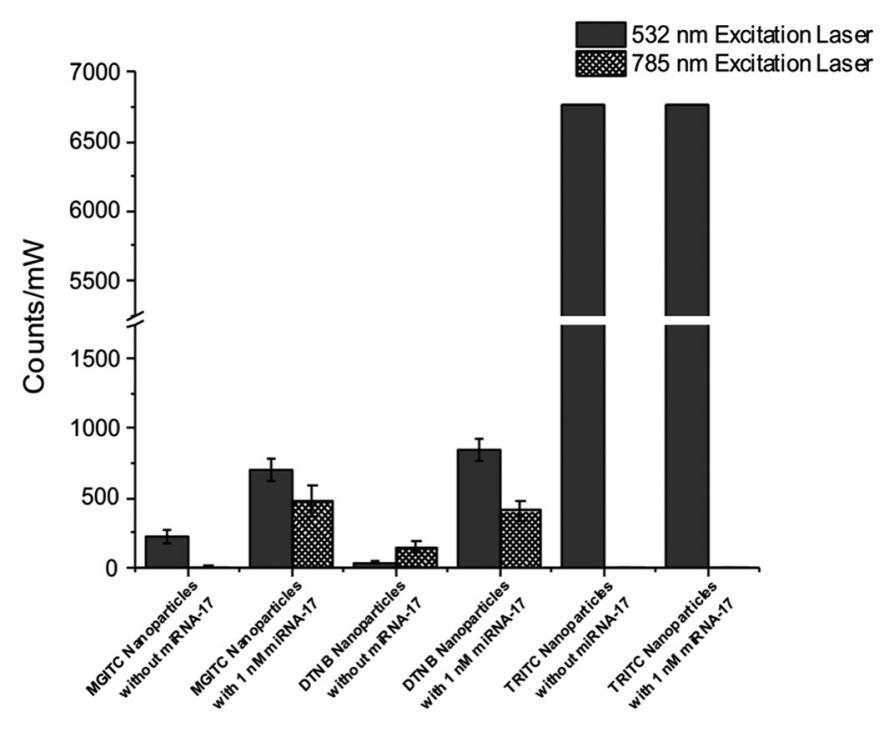

(a)

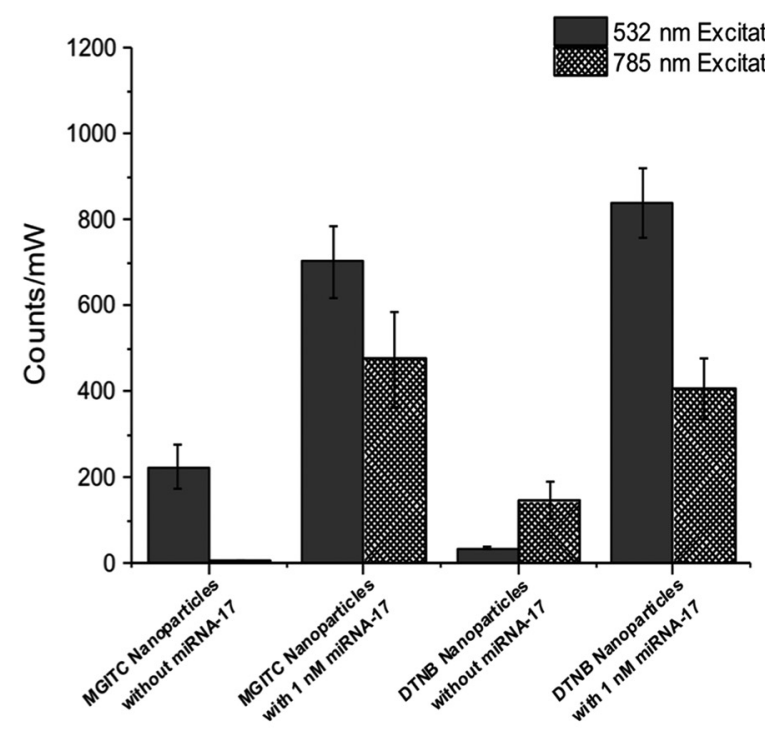

(b)

Fig. 7 Graphical representation of the relative Raman intensity examined at characteristic Raman peaks, normalized for the laser power and $\omega^{4}$ dependence of Raman scattering for (a) Raman reporter molecules (MGITC—peak at $1586 \mathrm{~cm}^{-1}$, DTNB_peak at $1343 \mathrm{~cm}^{-1}$, and TRITC) using both 532 and $785 \mathrm{~nm}$ excitation wavelengths and (b) an adjusted graph for MGITC and DTNB. 
that almost directly corresponds to the excitation wavelength of the incident laser. ${ }^{9}$ Our results indicate that signal enhancement can also be achieved at incident wavelengths beyond those that directly correspond to the dye's excitation wavelength. The data collected showed that if the laser's excitation wavelength was in the vicinity of the dye's excitation wavelength, signal enhancement still occurred. Namely, the DTNB assay (absorption maxima of $402 \mathrm{~nm}$ ) functioned properly for the 532-nm excitation, and the MGITC assay (absorption maxima of $620 \mathrm{~nm}$ ) performed better at $785 \mathrm{~nm}$. However, the TRITC (absorption maxima of $550 \mathrm{~nm}$ ) assay functioned poorly at $785 \mathrm{~nm}$ and was saturated at $532 \mathrm{~nm}$.

\subsection{Evaluation of Assay Specificity}

A noncomplimentary strand of miRNA, miRNA-34a-3p, was used as a negative control to test the specificity of the assay for defined target analyte, miRNA-17, and its effect on the baseline signal. The solution of the combined nanoparticle assay and noncomplimentary analyte was tested with all three Raman reporter molecules using an excitation laser of 532 (Fig. 8). The graph shows the resultant signal from the combination of the nanoparticle assay with miRNA34a-3p closely resemble that of the assay in PBS. Specifically, the maximum signal intensity of both the assay in PBS and the assay with miRNA34a-3p are about equal. Data also show that the addition of the noncomplimentary analyte did not alter the baseline signal.

\subsection{Comparison of Different Raman Reporter Molecules Using a Handheld Raman System}

Based on the results obtained using the benchtop Raman system, assays containing MGITC and DTNB were chosen for additional analysis in conjunction with the handheld Raman system. The volume of the sample needed for analysis was raised to $600 \mu \mathrm{L}$ to ensure sufficient volume was present within the 15 -mm glass vial, but the relative concentrations of assay components remained constant. Spectra were obtained using the handheld IDRaman mini for both the MGITC [Fig. 9(a)] and DTNB [Fig. 9(b)] dyes. Unlike the benchtop results, DTNB produced the strongest overall intensity signal for the IDRaman mini 2.0, with MGITC producing a significantly lower signal and exhibiting a higher noise floor. These results underscore the optical differences between the handheld and benchtop systems, implying that one cannot directly translate SERS systems from benchtop to handheld devices based solely on wavelength. Variations in the SERS response of the same two assays due to small differences in the filters, gratings, integration area, power, and source-along with the 10-fold change in resolution-are significant enough to warrant changing the Raman reporter

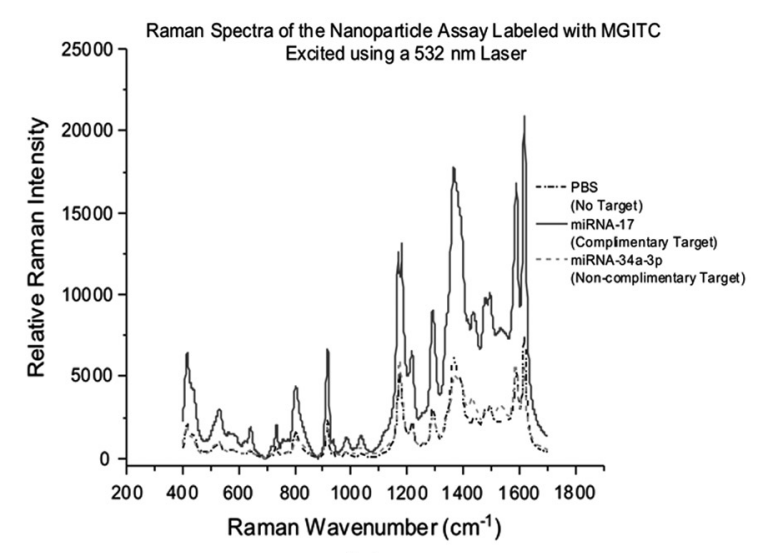

(a)

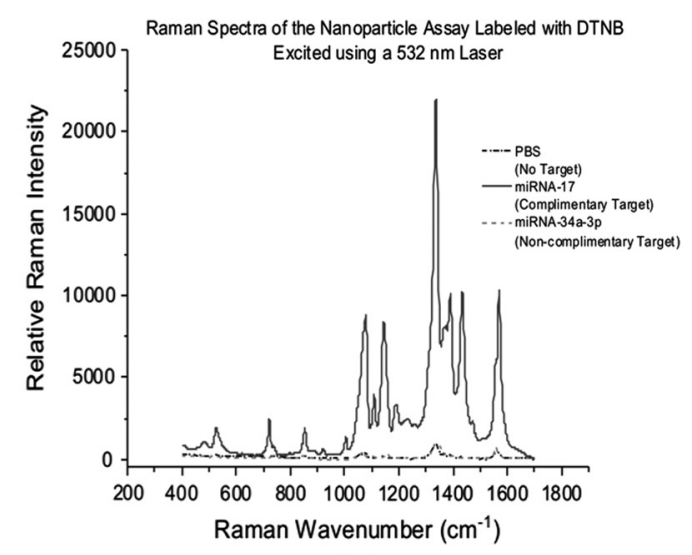

(b)

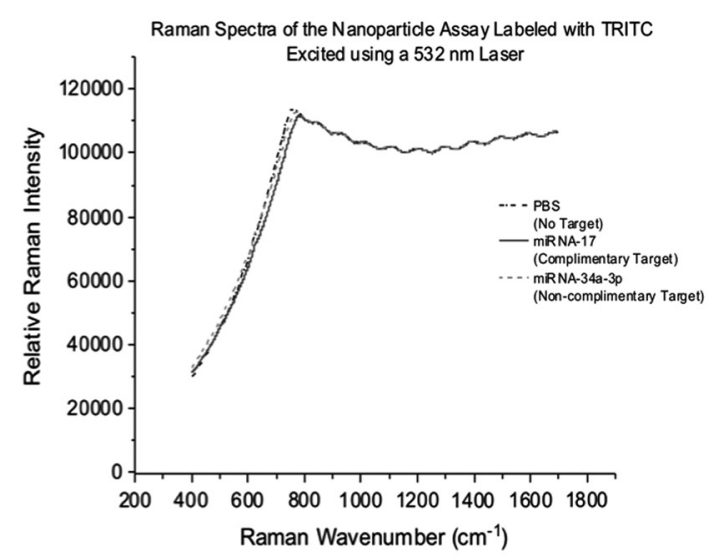

(c)

Fig. 8 Raman spectral data of the specificity of the assay when labeled with (a) MGITC, (b) DTNB, or (c) TRITC and excited using a 532-nm laser. 


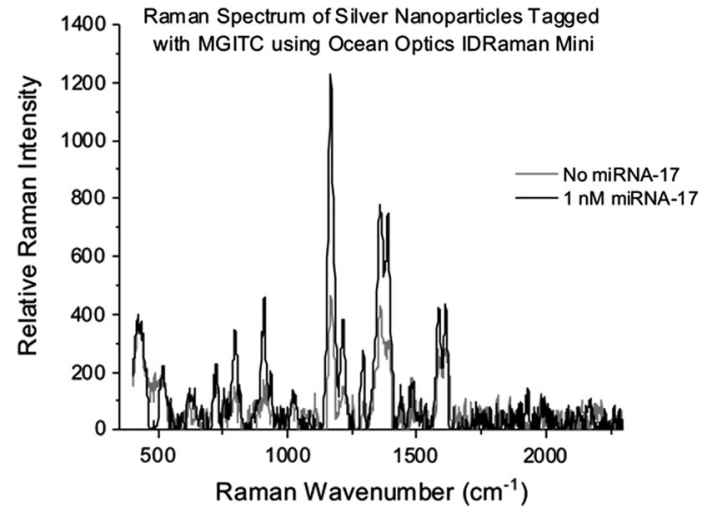

(a)

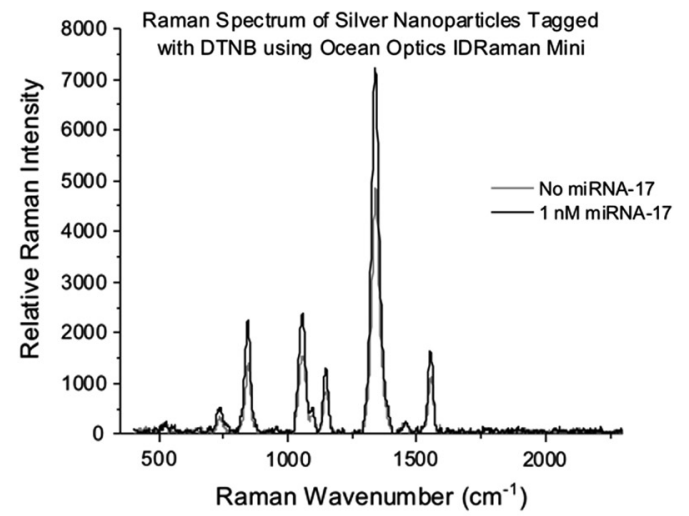

(b)

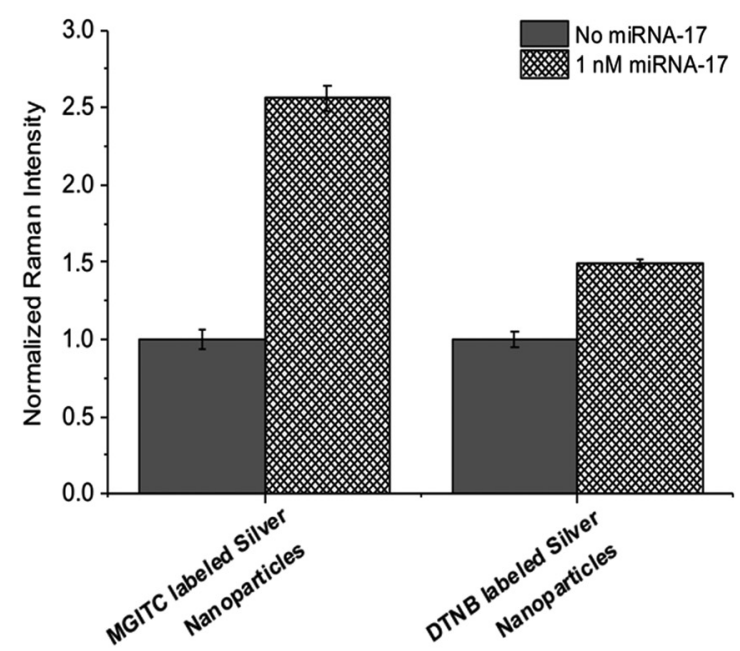

(c)

Fig. 9 Raman spectra for silver nanoparticles labeled with (a) MGITC and (b) DTNB in the presence of 1-nM target obtained using the handheld IDRaman mini; (c) comparison of normalized Raman intensity for MGITC (SERS Peak at $1586 \mathrm{~cm}^{-1}$ ) and DTNB (SERS peak at $1343 \mathrm{~cm}^{-1}$ ).

molecule entirely. Thus, using the same characteristic peaks as previously defined for MGITC $\left(1586 \mathrm{~cm}^{-1}\right)$ and DTNB $\left(1343 \mathrm{~cm}^{-1}\right)$, the maxima relative Raman intensity was determined and then normalized for further analysis. The normalized plot in Fig. 9(c) indicates that the assay labeled with MGITC showed the largest sensitivity, just as observed with the benchtop system for the NIR wavelength. Notably, there was a dramatic decrease in the sensitivity of the DTNB SERS signal with the assay when transitioning from the benchtop to handheld system due to the stronger signal with no target.

\section{Conclusion}

For both the benchtop and handheld Raman systems, nanoparticles, when combined with hybridized miRNA-DNA, provided pronounced SERS spectra when using MGITC and DTNB photoactive Raman molecules with 532- and 785-nm excitation lasers. Comparison with the benchtop assays synthesized with different Raman reporter molecules indicated that for the designed assay DTNB, with the addition of $1 \mathrm{nM}$ of target, was the highest performing Raman reporter molecule when combined with a 785-nm excitation laser, which is most often used in miniaturized Raman systems. Laser excitation wavelength heavily affected signal enhancement, which was smaller between target and no target when transitioning from the benchtop Raman system to the handheld Raman device. Additional data analysis indicated that signal enhancement is possible when using excitation lasers that do not directly coincide with the optical properties of the Raman reporter molecule. Nevertheless, enhancement is typically increased when excitation lasers most closely match the absorption maxima. Our results suggest that an understanding of plasmonics and the excitation and emission properties of assay components is necessary to facilitate the use of handheld Raman POC systems to advance clinical disease diagnosis in applications such as microRNA biomarker detection for early detection of preeclampsia.

\section{Disclosures}

The authors have no relevant financial interests and no potential conflicts of interest to disclose.

\section{Acknowledgments}

The study was supported by Texas A\&M Health Sciences Research Funding for interdisciplinary research. As per SPIE 
guidelines, the authors acknowledge that part of this work (proceedings number 10072-2) was presented at SPIE Photonics West in San Francisco, California, on January 30, 2017. This work was supported, in part, by funding from the National Science Foundation Engineering Research Center for Precise Advanced Technologies and Health Systems for Underserved Populations (PATHS-UP) (Award No. 1648451).

\section{References}

1. C. A. Holland and F. L. Kiechle, "Point-of-care molecular diagnostic systems_past, present and future," Curr. Opin. Microbiol. 8(5), 504-509 (2005).

2. M. Choudhury and J. E. Friedman, "Epigenetics and microRNAs in preeclampsia," Clin. Exp. Hypertens. 34(5), 334-341 (2012).

3. S. Meruvu, J. Zhang, and M. Choudhury, "Mono-(2-ethylhexyl) phthalate increases oxidative stress responsive miRNAs in first trimester placental cell line HTR8/SVneo," Chem. Res. Toxicol. 29(3), 430-435 (2016).

4. K. Kanasaki and R. Kalluri, "The biology of preeclampsia," Kidney Int. 76(8), 831-837 (2009).

5. C. W. Redman and I. L. Sargent, "Latest advances in understanding preeclampsia," Science 308(5728), 1592-1594 (2005).

6. D.-B. Chen and W. Wang, "Human placental microRNAs and preeclampsia," Biol. Reprod. 88(5), 130 (2013).

7. O. Biró, B. Nagy, and J. Rigó, "Identifying miRNA regulatory mechanisms in preeclampsia by systems biology approaches," Hypertens. Pregnancy 36(1), 90-99 (2017).

8. S. H. Yazdi, K. L. Giles, and I. M. White, "Multiplexed detection of DNA sequences using a competitive displacement assay in a microfluidic SERRS-based device," Anal. Chem. 85(21), 10605-10611 (2013).

9. T. Vo-Dinh et al., "SERS nanosensors and nanoreporters: golden opportunities in biomedical applications," WIREs Nanomed. Nanobiotechnol. 7(1), 17-33 (2015).

10. L. Guerrini and D. Graham, "Molecularly-mediated assemblies of plasmonic nanoparticles for surface-enhanced Raman spectroscopy applications," Chem. Soc. Rev. 41(21), 7085-7107 (2012).

11. G. McNay et al., "Surface-enhanced Raman scattering (SERS) and surface-enhanced resonance Raman scattering (SERRS): a review of applications," Appl. Spectrosc. 65(8), 825-837 (2011).

12. N. D. Israelsen, C. Hanson, and E. Vargis, "Nanoparticle properties and synthesis effects on surface-enhanced Raman scattering enhancement factor: an introduction," Sci. World J. 2015, 1-12 (2015).

13. D. Cialla et al., "Surface-enhanced Raman spectroscopy (SERS): progress and trends," Anal. Bioanal. Chem. 403(1), 27-54 (2012).

14. J. F. Betz et al., "Simple SERS substrates: powerful, portable, and full of potential," Phys. Chem. Chem. Phys. 16(6), 2224-2239 (2014).

15. K. Hering et al., "SERS: a versatile tool in chemical and biochemical diagnostics," Anal. Bioanal. Chem. 390(1), 113-124 (2008).

16. J. H. Granger et al., "Prospects for point-of-care pathogen diagnostics using surface-enhanced Raman scattering (SERS)," Chem. Soc. Rev. 45(14), 3865-3882 (2016).

17. Y. Wang, B. Yan, and L. Chen, "SERS tags: novel optical nanoprobes for bioanalysis," Chem. Rev. 113(3), 1391-1428 (2013).

18. N. Leopold and B. Lendl, "A new method for fast preparation of highly surface-enhanced Raman scattering (SERS) active silver colloids at room temperature by reduction of silver nitrate with hydroxylamine hydrochloride," J. Phys. Chem. 107, 5723-5727 (2003).

19. A. Michota and J. Bukowska, "Surface-enhanced Raman scattering (SERS) of 4-mercaptobenzoic acid on silver and gold substrates," J. Raman Spectrosc. 34(1), 21-25 (2003).

20. C. C. Lin et al., "A new protein A assay based on Raman reporter labeled immunogold nanoparticles," Biosens. Bioelectron. 24(2), 178-183 (2008).
21. E. Batista et al., "Using polycarbonate membranes as templates for the preparation of $\mathrm{Au}$ nanostructures for surface-enhanced Raman scattering," J. Nanosci. Nanotechnol. 9(5), 3233-3238 (2009).

22. V. Gubala et al., "Point of care diagnostics: status and future," Anal. Chem. 84(2), 487-515 (2012).

23. C.-W. Chang et al., "Fabrication of nano-indented cavities on Au for the detection of chemically-adsorbed DTNB molecular probes through SERS effect," J. Colloid Interface Sci. 358, 384-391 (2011).

24. J. Reichman, Handbook of Optical Filters for Fluorescence Microscopy, Chroma Technology Corp., Brattleboro, Vermont (1998).

25. B. Guven et al., "SERS-based direct and sandwich assay methods for mir-21 detection," Analyst 139(5), 1141-1147 (2014).

26. Y. Wang et al., "Direct detection of microRNA based on plasmon hybridization of nanoparticle dimers," Analyst 140(4), 1140-1148 (2015).

27. T. Donnelly, K. Faulds, and D. Graham, "Investigation of silver nanoparticle assembly following hybridization with different lengths of DNA," Part. Part. Syst. Char. 33(7), 404-411 (2016).

28. L. Barrett et al., "Stable dye-labelled oligonucleotide-nanoparticle conjugates for nucleic acid detection," Nanoscale 3(8), 3221-3227 (2011).

29. S. Mabbott et al., "From synthetic DNA to PCR product: detection of fungal infections using SERS," Faraday Discuss. 187, 461-472 (2016).

30. J. A. Dougan et al., "Enhanced oligonucleotide-nanoparticle conjugate stability using thioctic acid modified oligonucleotides," Nucleic Acids Res. 35(11), 3668-3675 (2007).

31. S. Schlücker, "Surface-enhanced Raman spectroscopy-concepts and chemical applications," Angew. Chem. Int. Ed. 53, 4756-4795 (2014).

32. S. Laing, K. Gracie, and K. Faulds, "Multiplex in vitro detection using SERS," Chem. Soc. Rev. 45(7), 1901-1918 (2016).

33. M. E. Pekdemir et al., "Ultrasensitive and selective homogeneous sandwich immunoassay detection by surface enhanced Raman scattering (SERS)," Analyst 137(20), 4834-4840 (2012).

34. T. Donnelly et al., "Silver and magnetic nanoparticles for sensitive DNA detection by SERS," Chem. Commun. 50(85), 12907-12910 (2014).

35. Thermo Scientific, "Thermo scientific DXR Raman microscope," https:// tools.thermofisher.com/content/sfs/brochures/DXR-RamanmicroscopeBR51343.pdf (2013).

36. Ocean Optics, "IDRaman mini," https://oceanoptics.com//wp-content/ uploads/IDRaman_Mini_Product_Sheet.pdf (2017).

37. Ocean Optics, "IDRaman mini 2.0," https://oceanoptics.com/product/ idraman-mini/ (2017).

38. Ocean Optics, "IDRaman reader product sheet," https://oceanoptics. com//wp-content/uploads/IDRaman_Reader_Product_Sheet.pdf (2017).

39. X. Zhang, M. R. Servos, and J. Liu, "Fast pH-assisted functionalization of silver nanoparticles with monothiolated DNA," Chem. Commun. 48(81), 10114-10116 (2012).

40. Ocean Optics, "IDRaman mini 2.0 installation and operation manual," https://oceanoptics.com/wp-content/uploads/IDRamanmini2manual.pdf (2015).

41. M. Li, S. K. Cushing, and N. Wu, "Plasmon-enhanced optical sensors: a review," Analyst 140(2), 386-406 (2015).

42. A. Kamińska et al., "Highly reproducible, stable and multiply regenerated surface-enhanced Raman scattering substrate for biomedical applications," J. Mater. Chem. 21(24), 8662-8669 (2011).

43. D. S. Grubisha et al., "Femtomolar detection of prostate-specific antigen - an immunoassay based on surface-enhanced Raman scattering and immunogold labels," Anal. Chem. 75(21), 5936-5943 (2003).

44. Y. K. Shrestha and F. Yan, "Determination of critical micelle concentration of cationic surfactants by surface-enhanced Raman scattering," RSC Adv. 4(70), 37274-37277 (2014).

45. C. C. Lin and C. W. Chang, "AuNPs@mesoSiO $\mathrm{S}_{2}$ composites for SERS detection of DTNB molecule," Biosens. Bioelectron. 51, 297-303 (2014).

Biographies for the authors are not available. 